\title{
A New Wireless Network Scheme for Spectrum Sensing in Cognitive Radio
}

\author{
Serkan Ozbay ${ }^{1}$, Ergun Ercelebi ${ }^{1}$ \\ ${ }^{I}$ Department of Electrical and Electronics Engineering, University of Gaziantep, \\ 27310 Gaziantep, Turkey \\ sozbay@gantep.edu.tr
}

\begin{abstract}
Spectrum sensing task is known to be one of the most important functionalities of a cognitive radio for dynamic spectrum access networks to be aware of the radio environment. Licensed user (primary user) detection has gained a great deal of interest recently on spectrum sensing strategies whereas unlicensed user (secondary user) detection must be a part of sensing with equal proportion of interest. This paper proposes a novel scheme that enables an efficient and robust spectrum sensing for both primary and secondary users detection. The proposed scheme is based on intentionally adding known noise at the sensing side. Then the sensing detector becomes insensitive to signal and noise types, and thus noise uncertainty problem is diminished. After sensing, the added noise is removed without affecting existing transmission on the channel. Although there have been several spectrum sensing methods, the proposed scheme is the optimal solution for spectrum sensing, to the best of our knowledge.
\end{abstract}

Index Terms-Cognitive radio, noise uncertainty, spectrum sensing, wireless network.

\section{INTRODUCTION}

Due to fast growing demand of wireless communications with a fixed spectrum allocation in finite radio sources, spectrum reuse applications motivate the recent researches. Spectrum reuse allows the unlicensed users to share the radio spectrum allocated to the licensed users when the radio spectrum is temporally vacant. Cognitive radio network is the key technology to improve the utilization of the spectrum efficiently. Allowing multiple users (primary user and secondary users) to share a radio channel needs true and fast algorithm for determining the status of the channel as occupied or idle. Meanwhile, spectrum share scheme must guarantee higher priority of primary users. That is, a secondary user must be only allowed to utilize the channel without causing harmful interference to the primary user. Thus spectrum sharing needs very fast user detection algorithms. Spectrum sensing concept covers the determination of the radio channel status, i.e., user detection on the radio source of interest. Hence spectrum reuse is not possible without spectrum sensing.

The objective behind spectrum sensing is to detect the presence or absence of users by monitoring a bandwidth of spectrum continuously. There have been numerous spectrum sensing strategies in literature serving different purposes and

Manuscript received February 14, 2015; accepted October 6, 2015. having advantages and also some drawbacks depending on the network and user characteristics. Hence there are still some factors motivating the researchers to study and making the sensing problem a challenging issue. First, in many practical wireless communication situations, there is no information about the primary user signal forms so sensing needs blind algorithms that operate without any prior knowledge on the primary signals and the channel. Second, the signal to noise ratio (SNR) at the sensing detector is very low thus the detector must be agile even if very low SNR values on the order of $-10 \mathrm{~dB}$ exist. Third, the communication environment may have some types of noises depending on the application, but there is always a noise uncertainty at the sensing detector; therefore detection must be good enough with noise uncertainty. Last the sensing time must be short enough, thus the sensing needs less complexities with faster operation.

Even if a high number of spectrum sensing techniques have been proposed in literature, sensing problem still has a lack of optimal solution. This paper addresses a novel approach usable for all channel environments.

The paper is organized as follows. In Section II, existing spectrum sensing techniques have been discussed with the advantages and limitations. Problem formulation and performance metric have been presented on Sections III and IV, respectively. Section V presents the proposed cognitive radio scenario and sensing algorithm. Performance analysis is provided in Section VI and finally the paper is concluded in Section VII.

\section{SPectrum SENSing Techniques on Cognitive Radio}

Depending on the different requirements of cognitive radio (CR) scenarios, spectrum sensing methods can be classified as: local sensing methods and cooperative sensing methods.

Local sensing methods contain transmitter detection and receiver detection using only one local sensing detector while cooperative sensing covers the multiple sensing detectors and relies on the information exchange among these detectors. To sense the channel availability at one time in CR communication architecture, monitoring the channel by detecting the primary user (PU) presence is the most widely used approach. PU can be either transmitter or receiver so the sensing can be both PU transmitter and PU receiver detection. 
Receiver detection methods assume that a PU is receiving data within the range of $\mathrm{CR}$ and that PU receiver is not passive while receiving the data from the PU transmitter. These methods use the fact that the receiver provides leakage of electromagnetic waves [1] and sensing detector can determine the presence of PU receiver by measuring the leakage power using radiometer (or known as energy detector). Since the receiver detection requires high sensitivity on the measurement of very small amount of leakage power, most local sensing methods focus on the PU transmitter detection.

Transmitter detection methods suppose that a PU transmitter sends data in the $\mathrm{CR}$ when communicating with the PU receiver(s) and the methods depend on the detection of primary transmitted signal based on the local measurements done by the local detection sensors. Generally proposed algorithms of this kind of detection are energy detection, matched filtering, cyclostationary detection, wavelet detection and detections based on signal statistics.

Energy detection algorithm makes measurements of energy in some portion of the CR in a finite time interval by a local detector and the presence of PU transmitted signal is determined by comparing the measurements to the predetermined threshold depending on the noise floor [2]. The energy detector based algorithm is known as the simplest, i.e., it has both low hardware cost and low computation complexity. It also has the advantage that it does not require a priori information about transmission data. Thus this method seems to be optimal. But this is only true on the condition that the probability density functions of the signals on both occupied and idle channel cases are perfectly known. This assumption generally fails when the noise uncertainty is high (knowledge of noise power is unknown). Hence accurate detection rate significantly falls down in lower SNR values due to the unknown noise variance.

Sensing problem on high noise uncertainty conditions can be overwhelmed with the matched filter detection. But this method highly depends on the prior knowledge about the PU transmitted signal [3]. It means transmitted signal to be detected must be deterministic to the sensing detector. Determination of presence of PU is achieved by correlating the unknown input signal with a time-reversed version of the template which is obtained from prior knowledge on signal transmission. Therefore this method can be thought as the optimal way by maximizing the detection rate in weak signals in a less processing time. On the other hand the use of matched filtering might be very rare since a priori information on PU transmitted signal may not be available in most of the CR scenarios.

Another well-known transmitter detection strategy is the cyclostationary detection which uses some statistical properties of PU transmitted signal [4]. In many wireless communications, transmitted signals inherently have some cyclostationary features including modulation type, modulating signal frequency or data rate. The presence or absence of the PU is determined by extracting the cyclostationary features of the received signal on the sensing detector and exploiting these specific features. If the cyclic autocorrelation function or cyclic spectrum obtained is above the threshold, the sensing algorithm marks the channel as occupied by PU; otherwise PU is absent. As in the matched filter detection, this method is more robust to noise uncertainty than the energy detection. It provides better detection performance under low SNR regime. However cyclostationary detection is not a blind sensing algorithm so that it needs a prior knowledge of the PU signal. It is the main drawback of the method and also it is more complex to implement and requires high computations and more processing time.

The wavelet based detection algorithm uses wavelet transform to detect the PU users. Since the wavelet transforms help to show irregularities on the power spectrum of the signals, the wavelet based sensing algorithm uses this fact to determine the edges of PU in a wide-band spectrum in the frequency domain [5]. Wavelet based signal detection over wide-band communications can thus perform better spectrum sensing. As well as the advantageous use on wideband communications, the main drawback of the method is that it requires very high sampling rate. It needs high sampling rates because of the large bandwidth characteristics.

Popular and recent detection algorithms use some statistical information about transmitted signal and noise in the wireless channel. One way of detecting is to estimate the second-order statistics of the received signal on sensing detector and make decisions about the presence of the PU according to these estimates [6]. It is known that the statistical covariance of the transmitted wireless signals and the noise on the channel are generally different from each other and this fact can be used as a classifier of presence and absence of the PU in a CR. Well-known methods which work in this manner are covariance based detection, eigenvalue detection [7]-[8] or eigenvector detection [9]. These approaches give the advantages that they operate without knowledge of signal and noise power and they require no a priori information. However these algorithms have higher computational complexities. Some goodness of fit (GoF) including Anderson-Darling based [10], Kolmogorov-Smirnov [11] or Jarkur-Bera tests [12] have been recently proposed as the sensing algorithm. A GoF test is a statistical method describing how well it fits a set of observations or measurements. These algorithms are said to be robust to noise uncertainty but they have longer processing time because of their high computational cost.

The detection performance of spectrum sensing algorithms significantly degrades due to destructive channel conditions such as fading and shadowing. Fortunately, performance degradation can be improved by the cooperative sensing among sensing detectors [2], [13]. The main idea on cooperative sensing is to share the sensors individual sensing information by using the spatial diversity of local sensors and making a fused decision which is more accurate than the individual decisions. Cooperative sensing strategies can be classified into three main categories: centralized, distributed, and relay-assisted which differ from each other by how cooperating users share/exchange the sensing data in the network [14]-[18]. The main pros of cooperative sensing are that the hidden node problems are reduced and more accurate detection performance is 
achieved. On the other hand it requires complex control channel and complex synchronization between distributed sensors.

In addition to the sensing methods summarized above, some different approaches have been proposed mainly as multiple antenna sensing and multiple-input and multiple output (MIMO) spectrum sensing. In multiple antenna spectrum sensing, sensing detector has more receiving antennas to monitor the channel [19]. Although use of more antennas increases the accuracy of detection, its implementation requires more computational cost and hardware cost. Similar to the multiple antenna technique, MIMO sensing model has multiple antennas at both receiver and transmitter part of the communication system. This sensing model enables wideband sensing as the number of antennas increases. However, it requires complex control mechanisms and it has synchronization problems.

\section{PROBLEM FORMULATION}

The problem of spectrum sensing is simply to determine whether a bandwidth of spectrum (channel) is occupied or idle at a time. In other words, the presence or absence of users in a bandwidth of interest is identified with the sensing approach. In mathematical notation, it is described by a binary hypothesis-testing problem with two hypotheses $H_{0}$ and $H_{1}$ :

$$
\begin{gathered}
H_{0}: \text { Channel is temporally vacant, } \\
H_{1}: \text { Channel is occupied by user }(s) .
\end{gathered}
$$

Thus the spectrum sensing problem is to decide between these hypotheses:

$$
\begin{gathered}
H_{0}: y(t)=n(t), \\
H_{1}: y(t)=x(t)+n(t),
\end{gathered}
$$

where $y(t)$ represents the signal observed at the local CR sensing detector, $x(t)$ denotes the received signal coming from channel user(s), and $n(t)$ represents the noise signal on the wireless channel.

The given formulation is valid for local sensing strategies. On cooperative sensing, the individual sense decisions are fused together to form a final decision and fusion process may change according to the various algorithms.

In spectrum sensing problem the testing detects whether the observation $y$ was provided under $H_{0}$ or $H_{1}$. Generally this process is carried out by comparing the testing value taken from $y$ to a predefined threshold. If the test value is greater than the threshold, it is assumed to have $H_{1}$ hypothesis; otherwise $H_{0}$.

\section{PERFormance Metric}

A comprehensive performance of spectrum sensing algorithms is mostly evaluated in terms of the following parameters: Accuracy, Complexity and Sensing Duration.

Determination of accuracy of spectrum sensing is quantified with respect to the statistical terms: probability of accurate detection, probability of detection, probability of missed detection and probability of false alarm given by the following definitions:

Probability of accurate detection

$$
P_{a d}=\operatorname{Pr}\left\{\text { Decision }=H_{1} \mid H_{1}\right\}+\operatorname{Pr}\left\{\text { Decision }=H_{0} \mid H_{0}\right\} .
$$

Probability of detection

$$
P_{d}=\operatorname{Pr}\left\{\text { Decision }=H_{1} \mid H_{1}\right\} .
$$

Probability of false alarm

$$
P_{f}=\operatorname{Pr}\left\{\text { Decision }=H_{1} \mid H_{0}\right\}
$$

Probability of missed detection

$$
P_{m}=\operatorname{Pr}\left\{\text { Decision }=H_{0} \mid H_{1}\right\} .
$$

Sensing accuracy is generally determined by the so-called receiver operating characteristic (ROC) curves. A ROC curve is either the plot of probability of accurate detection versus the probability of false alarm or the plot of probability of missed detection versus probability of false alarm.

Accuracy highly depends on the noise thus robustness to different kind of noises, noise uncertainty, and SNR dependence must be determined as part of the evaluation.

Complexity covers both hardware complexity and processing (or computational) complexity and sensing methods must have reasonable complexity values. The processing complexity heavily affects the sensing time and thus it adds extra delay to the algorithm.

Finally sensing duration is one of the most important points of spectrum sharing architectures. Secondary users must vacate the channel as soon as possible if the licensed users exist. This is so important because of protecting the licensed user rights and thus avoiding interference.

\section{PROPOSED COGNITIVE RADIO SCENARIO AND SPECTRUM SENSING}

To meet all the requirements of the performance metric defined, an algorithm robust to noise uncertainty, independent from signal type and format, insensitive to noise types, accurate under low SNR values, simple to implement and finally fast and less complex must be proposed. To do so, a novel cognitive radio scenario is addressed to cover all necessities.

In the scenario, a wireless regional area network (WRAN) where it has nearly constant background noise at all points in the region is proposed. It is given that the lack of knowledge about the noise power (noise uncertainty) degrades sensing detection performance. We propose to keep the noise constant. Noise, which has known characteristics, will be emitted to the wireless region and its level will be kept constant. In the proposed scenario, this process can be considered as extra noise addition at first inspection. But the known noise addition can be eliminated by the users. The critical issue is the establishment of a constant and known 
noise in a wireless region. Thus the problems coming from noise uncertainty and different kind of noise types will be diminished because of known dominant noise characteristics. We do no longer care about the actual noise. Simple detection algorithms such as energy detection based and matched filtering based algorithms could be efficiently used in the proposed scenario.

Many spectrum sensing strategies focus on primary user detection. On the other hand, the heart of the spectrum sharing via the cognitive radio is to use the channel with both the PUs and unlicensed SUs. As expected, the number of SUs at a time can be more than one. Thus in the case where the PUs are inactive and specific SUs are active, the channel can be monitored by the other opportunistic SUs. Thus the detection algorithms must be sensitive to not only known PU signal but also to unknown SU signals. Matched filtering and cyclostationary detection algorithms fail on those situations. Thus a blind algorithm which does not require a priori knowledge about the users must be proposed.

With the proposed cognitive radio scenario, an optimal spectrum sensing algorithm is defined. A local sensing detector makes measurements of energy in some portion of the $\mathrm{CR}$ at first as in the energy detection algorithm and then since the dominant noise is known, the correlation of the measured signal samples with the predefined background noise signal samples is used for determining the presence of the user like the matched filtering. Thus the optimal way of spectrum sensing is obtained. It takes the simplicity advantage of energy detection and noise uncertainty advantage of matched filtering.

Now the spectrum sensing problem is to decide between these hypotheses:

$$
\begin{gathered}
H_{0}: y(t)=n(t)+n_{\text {added }}(t), \\
H_{1}: y(t)=x(t)+n(t)+n_{\text {added }}(t) .
\end{gathered}
$$

After sensing process, the added signal is subtracted from the transmitted data by the help of known characteristic. Thus it does not produce any distortion problem.

\section{PERFORMANCE ANALYSIS}

\section{A. Experimental Setup}

In order to evaluate the performance of the proposed network scheme, we have used a test bed for simulating the network and implementing the sensing algorithm. The test bed contains Universal Software Radio Peripheral 2 (USRP2) boards attached with computers with GNU Radio software. USRP2 is a flexible low-cost cognitive radio platform developed by Ettus Research [20]. It provides radio front-end functionalities with an FPGA. The USRP2 allows making signal processing operations on a computer and general purpose tasks including decimation, interpolation, digital up-down conversions in it. GNU Radio is a free and open source toolkit providing a library of signal processing blocks for implementing the cognitive radio applications [21].

We used real primary signals for the performance analysis. Real signal samples are produced by a transmitter design which is a combination of GNU Radio and USRP2. The transmitter produced an OFDM signal with 40 sub carriers with BPSK modulation at a centre frequency of $435 \mathrm{MHz}$ (a frequency band on ISM) with a $1 \mathrm{MHz}$ bandwidth. For the noise addition intentionally to simulate the proposed network scheme, USRP2+GNU Radio platform is also used to generate noise samples. The spectrum sensing detector is formed using GNU Radio and USRP2 as well. The detector is responsible for collecting data on the channel and deciding the channel status as vacant or occupied by users.

\section{B. Results}

Generated signals for simulating the users on the channel are OFDM-based and it is assumed that they are noise-free. The proposed algorithm is tested with the noise-free channel. Moreover, to analyse the performance of the proposed network scheme in a noisy environment, non-fading noisy channel (additive white Gaussian channel), Rician fading channel and Nakagami fading channel are simulated and the performance is evaluated.

Additive white Gaussian channel (AWGN) is a channel model in which the only impairment to communication is a linear addition of wideband or white noise with a constant spectral density and a Gaussian distribution of amplitude. AWGN is a good model to simulate background noise of the channel under study, in addition to multipath, terrain blocking, interference, ground clutter and self-interference that modern radio systems encounter in terrestrial operation.

According to the nature of radio wave propagation, the radio signals may be affected by the phenomena of path loss variance with distance, shadowing (or long-term fading), and multipath (or short-term) fading. Shadowing and multipath fading effects, except the path loss which only depends on the distance, can be statistically described by fading models. Since a number of varieties of fading environments exist, several statistical distributions have been proposed for fading channels including Rician/Rayleigh fading environments. In Rayleigh fading models, it is assumed that the signal strength will vary randomly as it passes through the fading channel, i.e., the signal fades. If a dominant line of sight exists between the transmitter and receiver, Rician fading models become more realistic. Thus, in a wireless communication environment, where a direct line of sight accompanied by the diffused signal component occurs, Rician fading model based on the Rician distribution has been proposed to be a more accurate model for the fading statistics [22].

In addition to Rayleigh and Ricean distributions to model fading channels, Nakagami fading distribution is used to model some fading environment. Nakagami fading is said to have good fit to empirical fading data [23]. The Nakagami fading distribution was first proposed by Nakagami to simulate rapid fading in high frequency channels. Nakagami fading model is appropriate on the condition when the multipath scattering with relatively large delay-time spreads occurs on the channel.

To simulate the AWGN non-fading channel, Rician fading channel and Nakagami fading channel, we produced noise 
samples based on Gaussian distribution, Rician distribution and Nakagami-m distribution, respectively. Hence, in a noisy environment simulation, when there is no user signal transmission, the only signal on the channel is noise; and when the signal transmission exists on the channel, the sensed samples are the sum of primary signal and noise.

To determine the validity of the proposed network scheme and observe the performance of the proposed sensing approach, some measurements are carried out by a sensing detector with no signal and signal transmission case. On both situations, the intentionally added noise is produced thus the measured samples include those noise samples.

Table I shows the proposed sensing algorithm performance comparison with some existing blind algorithms such as energy detection (ED), cooperative energy detection (CED), Anderson-Darling based detection (AD) and Kolmogorov-Smirnov based detection (KS). The samples are used directly without adding any noise samples. Here the target probability of false alarm $P_{f}$ value is set to

0.1 and the measurements are done on the average $-8 \mathrm{~dB}$ SNR value. Totally 100,000 sets each of which includes 200 samples have been used in the performance analysis.

TABLE I. PROBABILITY OF DETECTION $P_{d}$ VALUES OF VARIOUS SENSING METHODS UNDER NOISE-FREE ENVIRONMENT AT $P_{f}=0.1$ AND AVERAGE $S N R=-8 d B$

\begin{tabular}{|c|c|c|c|c|c|}
\hline Detector & $\begin{array}{c}\text { Proposed } \\
\text { Spectrum } \\
\text { Sensing(PSS) } \\
\text { algorithm }\end{array}$ & ED & CED & AD & KS \\
\hline$P_{d}$ & 0.984 & 0.822 & 0.940 & 0.520 & 0.904 \\
\hline
\end{tabular}

The Receiver Operating Characteristic (ROC) curves of the proposed scheme algorithm and some existing sensing algorithms are depicted in Fig. 1-Fig. 4.

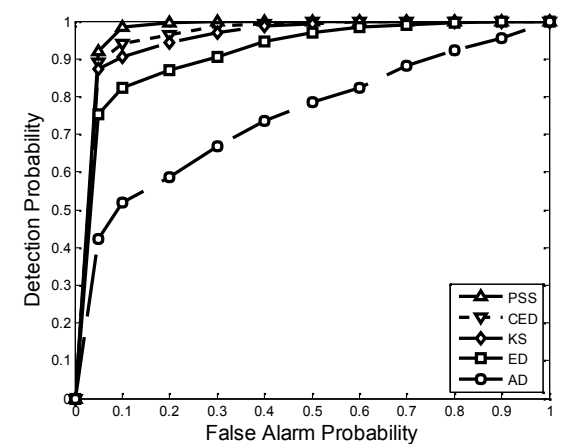

Fig. 1. ROC curves at $S N R=-8 d B$ under noisefree channel.

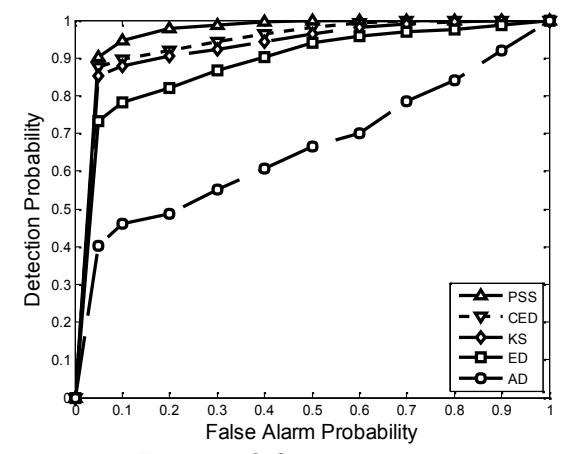

Fig. 2. ROC curves at $S N R=-8 d B$ under AWGN channel.
The measurements on the sensing detector are done in approximately $-8 \mathrm{~dB}$ SNR values. In each performance evaluation of the algorithm, the threshold is set to zero initially and then increased gradually step by step to see the corresponding probability of detection and probability of false alarm values.

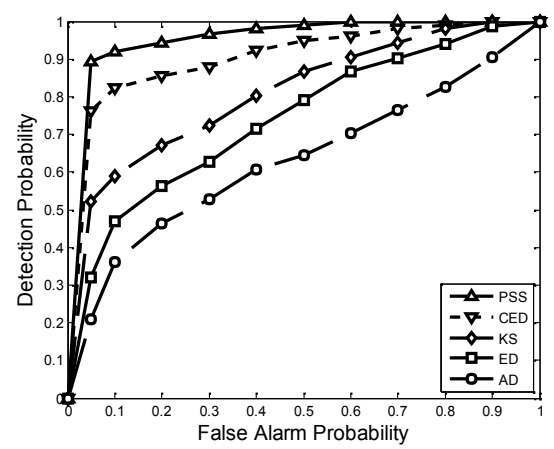

Fig. 3. ROC curves at $S N R=-8 d B$ under Rician fading channel.

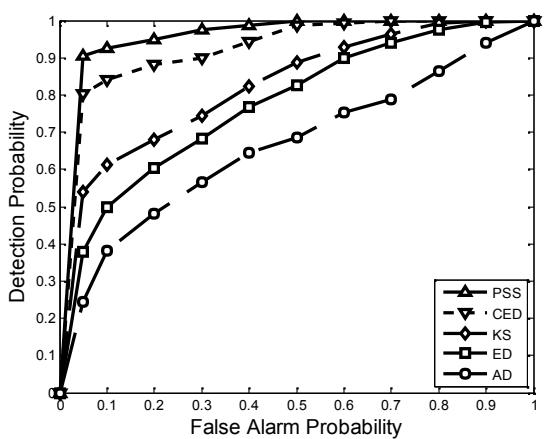

Fig. 4. ROC curves at $S N R=-8 d B$ under Nakagami fading channel.

To see the performance of the proposed algorithm under lower SNR values and compare to the existing algorithms, the probabilities of detection $P_{d}$ values versus different SNR values are shown in Fig. 5-Fig. 8. The target probabilities of false alarm $P_{f}$ values are set to 0.1 .

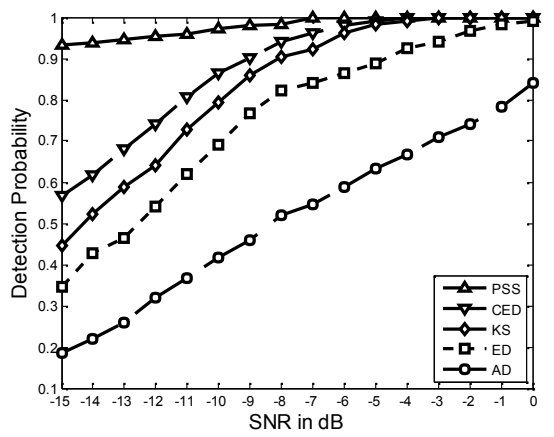

Fig. 5. The graph of $P_{d}$ versus SNR values under noisefree channel.

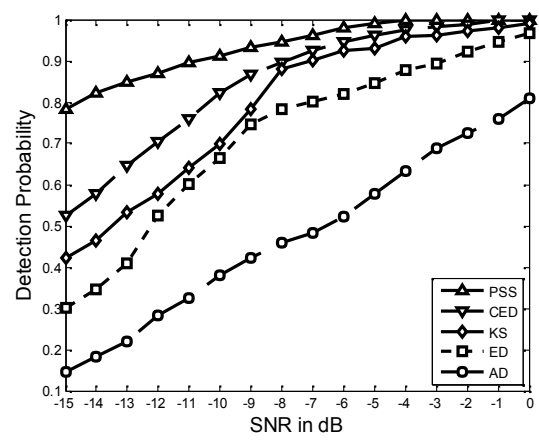

Fig. 6. The graph of $P_{d}$ versus SNR values under AWGN channel. 


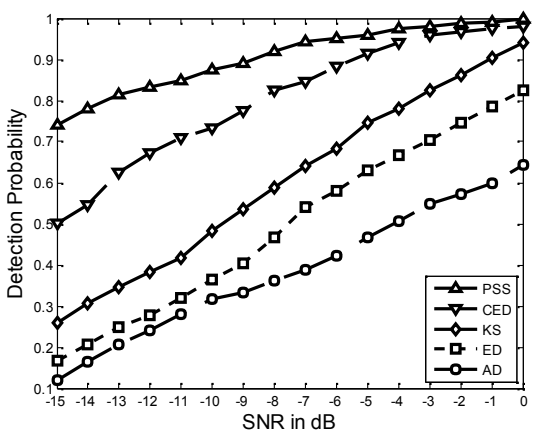

Fig. 7. The graph of $P_{d}$ versus SNR values under Rician fading channel.

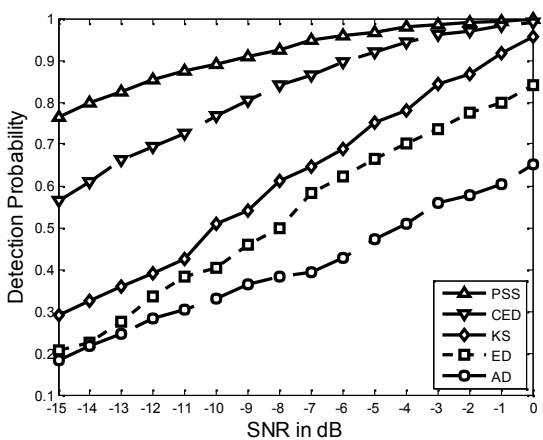

Fig. 8. The graph of $P_{d}$ versus SNR values under Nakagami fading channel.

\section{CONCLUSIONS}

In this paper, we proposed a new cognitive radio scheme focusing on spectrum sensing capability and investigated its performance under various noisy and fading environments. The proposed algorithm mainly addresses the sensing problem of secondary users as well as the primary users without any prior knowledge. The given spectrum sensing algorithm performance analysis has been conducted using real data and the experimental observations have been obtained. The experimental results show that the proposed algorithm outperforms the existing methods and is robust on noisy environments. It is also shown that the proposed algorithm is very effective and robust on the Rician and Nakagami fading models. By the novel method presented here, $90 \%$ true detection performance is achieved on the orders of nearly $-10 \mathrm{~dB}$ SNR values.

\section{REFERENCES}

[1] B. Wild, K. Ramchandran, "Detecting primary receivers for cognitive radio applications", 1st IEEE Int. Symposium on New Frontiers in Dynamic Spectrum Access Networks, Baltimore, MD, USA, 2005, pp. 124-130. [Online]. Available: http://dx.doi.org/10.1109/dyspan. 2005.1542626

[2] K. B. Letaief, W. Zhang, "Cooperative communications for cognitive radio networks", Proceedings of IEEE, vol. 97, no. 5, pp. 878-893, 2009. [Online]. Available: http://dx.doi.org/10.1109/JPROC. 2009.2015716

[3] T. Yucek, H. Arslan, "A survey of spectrum sensing algorithms for cognitive radio applications", IEEE Comm. Surveys and Tutorials, vol. 11, no. 1, pp. 116-130, 2009. [Online]. Available: http://dx.doi.org/10.1109/SURV.2009.090109

[4] S. Haykin, D. J. Thomson, J. H. Reed, "Spectrum sensing for cognitive radio", in. Proc. of IEEE, vol. 97, no. 5, pp. 849-877, 2009. [Online]. Available: http://dx.doi.org/10.1109/JPROC.2009.
2015711

[5] Z. Tian, G. B. Giannakis, "A wavelet approach to wideband spectrum sensing for cognitive radios", 1st Int. Conf. Cognitive Radio Oriented Wireless Networks and Communications, Mykonos, Greece, 2006, pp. 1-5. [Online]. Available: http://dx.doi.org/10.1109/crowncom. 2006.363459

[6] E. Axell, E. G. Larsson, "Optimal and sub-optimal spectrum sensing of OFDM signals in known and unknown noise variance," IEEE Journal on Sel. Areas in Comm., vol. 29, pp. 290-304, 2011. [Online]. Available: http://dx.doi.org/10.1109/JSAC.2011.110203

[7] Y. Zeng, Y.-C. Liang, "Eigenvalue-based spectrum sensing algorithms for cognitive radio", IEEE Trans. on Comm., vol. 57, no. 6, pp. 1784-1793, 2009. [Online]. Available: http://dx.doi.org/ 10.1109/TCOMM.2009.06.070402

[8] Y. Zeng, Y.-C. Liang, "Spectrum-sensing algorithms for cognitive radio based on statistical covariances", IEEE Trans. on Vehicular Technology, vol. 58, no. 4, pp.1804-1815, 2009. [Online]. Available: http://dx.doi.org/10.1109/TVT.2008.2005267

[9] P. Zhang, R. Qiu, "GLRT-based spectrum sensing with blindly learned feature under rank-1 assumption", IEEE Trans. on Comm. vol. 61, no. 1, pp. 87-96, 2013. [Online]. Available: http://dx.doi.org/ 10.1109/TCOMM.2012.100912.120162

[10] H. Wang, E. Yang, Z. Zhao, W. Zhang, "Spectrum sensing in cognitive radio using goodness of fit testing", IEEE Trans. on Wireless Comm., vol. 8, no. 11, pp. 5427-5430, 2009. [Online]. Available: http://dx.doi.org/10.1109/TWC.2009.081586

[11] G. Zhang, X. Wang, Y. Liang, J. Liu, "Fast and robust spectrum sensing via Kolmogorov-Smirnov test", IEEE Trans. on Wireless Comm., vol. 58, no. 12, pp. 3410-3416, 2010. [Online]. Available: http://dx.doi.org/10.1109/TCOMM.2010.11.090209

[12] L. Lu, H. Wu, S. S. Iyengar, "A novel robust detection algorithm for spectrum sensing", IEEE Journal on Sel. Areas in Comm., vol. 29, no. 2, pp. 305-315, 2011. [Online]. Available: http://dx.doi.org/ 10.1109/JSAC.2011.110204

[13] I. F. Akyildiz, B. F. Lo, R. Balakrishnan, "Cooperative spectrum sensing in cognitive radio networks: a survey", Physical Communication, vol. 4, no. 1, pp. 40-62, 2011. [Online]. Available: http://dx.doi.org/10.1016/j.phycom.2010.12.003

[14] J. Unnikrishnan, V. V. Veeravalli, "Cooperative sensing for primary detection in cognitive radio", IEEE Journal of Selected Topics in Signal Processing, vol. 2, no. 1, pp. 18-27, 2008. [Online] Available: http://dx.doi.org/10.1109/JSTSP.2007.914880

[15] Z. Li, R. R. Yu, M. Huang, "A cooperative spectrum sensing consensus scheme in cognitive radio", in Proc. IEEE INFOCOM, 2009, pp. 2546-2550. [Online]. Available: http://dx.doi.org/10.1109/ infcom.2009.5062184

[16] G. Ganesan, Y. G. Li, "Cooperative spectrum sensing in cognitive radio-part I: two user networks", IEEE Trans. on Wireless Comm. vol. 6, no. 6, pp. 2204-2213, 2007

[17] G. Ganesan, Y. G. Li, "Cooperative spectrum sensing in cognitive radio-part II: multiuser networks", IEEE Trans. on Wireless Comm. vol. 6, no. 6, pp. 2214-2222, 2007. [Online]. Available: http://dx.doi.org/10.1109/TWC.2007.05776

[18] W. Zhang, K. B. Letaief, "Cooperative spectrum sensing with transmit and relay diversity in cognitive radio networks", IEEE Trans. on Wireless Comm., vol. 7, no. 12, pp. 4761-4766, 2008 [Online]. Available: http://dx.doi.org/10.1109/T-WC.2008.060857

[19] A. Taherpour, M. N.-Kenari, S. Gazor, "Multiple antenna spectrum sensing in cognitive radios", IEEE Trans. on Wireless Comm., vol. 9, no. 2, pp. 814-823, 2010. [Online]. Available: http://dx.doi.org/ 10.1109/TWC.2009.02.090385

[20] Ettus Research LLC: Universal Software Radio Peripheral 2. [Online]. Available: http://www.ettus.com

[21] E. Blossom, Exploring GNU Radio. [Online] Available: http://www.gnu.org

[22] Y.-D. Yao, A. U. H. Sheikh, "Outage probability analysis for microcell mobile radio systems with cochannel interferers in Rician/Rayleigh fading environment", Electronics Letters, vol. 26, no. 13, pp. 864-866, 1990. [Online]. Available: http://dx.doi.org/10.1049/el:19900566

[23] H. Suzuki, "A statistical model for urban multipath", IEEE Trans. on Comm., vol. 25, no. 7, pp. 673-680, 1977.

[Online]. Available: http://dx.doi.org/10.1109/TCOM.1977.1093888 